\title{
Field Study on the EMDR Integrative Group Treatment Protocol for Ongoing Traumatic Stress with Female Survivors of Exploitation, Trafficking and Early Marriage in Dhaka, Bangladesh
}

\author{
Kelly Smyth-Dent ${ }^{1 *}$, Sarah Frances Walsh ${ }^{2}$ and Sadie Smith ${ }^{3}$ \\ ${ }^{1}$ Department of Research, United States \\ ${ }^{2}$ Liberty University Instructor, United States \\ ${ }^{3}$ Private Practice Clinician, United States
}

Submission: July 22, 2020; Published: September 04, 2020

*Corresponding author: Kelly Smyth-Dent, Department of Research, Scaling Up, United States

Abstract

The main objective of this study was to evaluate the effectiveness of the EMDR-Integrative Group Treatment Protocol for Ongoing Traumatic Stress (EMDR-IGTP-OTS) in reducing posttraumatic stress disorder (PTSD), depression, and anxiety symptoms with females in Dhaka, Bangladesh (and the surrounding areas) who have survived exploitation, trafficking and/or early marriage (under 15 years old). A total of 27 females were treated simultaneously with intensive EMDR therapy (six group sessions during two consecutive days, three times per day). Each of the treatment group participants received an average of six hours of EMDR therapy. EMDR-IGTP-OTS treatment focused only on the distressing memories related to their experience of exploitation, trafficking and/or early marriage. No adverse effects were reported during treatment, post-treatment, or at 90-day follow-up assessment. The Hospital Anxiety and Depression Scale (HADS) and the Post-traumatic Stress Disorder Checklist for DSM-5 (PCL5) were used as pre- and post-treatment assessments measuring each client's anxiety, depression, and PTSD symptoms, respectively. The ANOVA results of the HADS-anxiety scores $F(2,42)=3.62, p=.035$ and the HADS-depression scores $F(1.54,32.34)=0.96, p>.05$ show that the measures were significantly different between tests. The statistical analysis of the PCL-5 measures was also significantly different between tests, $F(2,42)=8.45, p=.001$. The study results show that the EMDR-IGTP-OTS could be an important component of a multidisciplinary approach to reduce or eliminate PTSD, depression and anxiety symptoms of participants. More research is needed to address the mental, social and health-care needs of this vulnerable population.

Keywords: EMDR therapy; EMDR-IGTP-OTS; Group therapy; Trauma exposure; Child bride; Early marriage; Exploitation; Trafficking

\section{Introduction}

Six hundred and fifty million girls and women alive today were married before their 18th birthday [1]. In Bangladesh, between the years 2010 and 2017, 59\% total of women were married before age 18 , with $22 \%$ of the population being married under the age of 15 [1,2]. Despite considerable social and economic progress in Bangladesh, child marriage remains the norm [3]. The negative effects of child marriage include spouse violence and controlling behaviors [4], suicidal thoughts and attempts [5], mental health issues [6], infant and child mortality and other health issues [7] and low educational attainment [8]. These women may suffer from the symptoms of Post-Traumatic Stress Disorder (PTSD), anxiety disorders, and depressive disorders with very little research to show what treatment or resources would be helpful for them. Not only are these women exploited in child marriage and trafficking, but they are also neglected by researchers. This study seeks to address the mental health needs of vulnerable girls and women in Bangladesh who were in child marriages, trafficked and/or exploited.

\section{Study participant's background and definition of terms}

The backgrounds of the women in this study are diverse. They all come from vulnerable, impoverished or dysfunctional homes, often leading to ongoing or severe trauma, including one or more of the following: family breakdown, homelessness, child marriage, 
sex trafficking, rape, imprisonment, abandonment by spouse, death of spouse or parent, child sexual abuse, or formal/informal prostitution. For the sake of narrowing down the definition in this publication, the authors have focused the literature review on the effects of child marriage. Additionally, the terms used most throughout this paper will be child marriage, trafficking and exploitation - as these three words encompass the experience of all the women in the study.

\section{Background of the problem}

Children sold into marriage attain less education, have lower participation in the labor force, face higher risk of death in childbirth, and are more likely to experience domestic violence $[9,10]$. Likewise, these women's children tend to be less educated and suffer poorer health [8]. In some countries and villages where parents strongly prefer sons to daughters, parents may seek to dispose of their unwanted daughters through child-bride marriage [9]. Parents tend to rush the marriage of their daughter if she has a younger sister [11]. There may also be social norms which requires parents to marry off their daughters before choosing a bride for their son(s) [11]. Little information exists on the mental health implications of and possible solutions for child marriage [5]. Bangladesh has the highest prevalence of Intimate Partner Violence (IPV) and very early child marriage (before age 15); Intimate partner violence (IPV) includes physical, sexual, or psychological aggression, stalking, or coercion by a current or former intimate partner [10].

Existing studies have been done on the educational, healthrelated, and violence-related issues of the population of child brides [3-10]. Similarly, many EMDR studies have been conducted on populations who have experienced PTSD and sexual abuse [12]. However, very little research has been done on the treatment of the mental health of these women. The problem therein lies in the lack of research and treatment options to serve this large and underserved population.

\section{EMDR therapy}

Eye movement desensitization and reprocessing (EMDR) is an evidence-based, psychological treatment that was initially developed to treat PTSD [12]. EMDR therapy has been found efficacious in treating chronic posttraumatic stress disorder [1315]. Consequently, EMDR therapy is recommended as a treatment of choice for children, adolescents and adults by the World Health Organization treatment guidelines for PTSD [16]. EMDR is an effective, psychological intervention for treating post-traumatic stress disorder (PTSD) [14] in both adults and children [15]. EMDR therapy is founded on the understanding of the Adaptive Information Processing (AIP) theoretical model which posits that memory networks are the foundation of pathology and overall health and wellness [12]. The AIP model explains that psychopathology is primarily caused by memories of traumatic or adverse life experiences which has been inadequately processed and maladaptively stored in a "state-specific form, meaning that the information acquired at the time of the event -images, sounds, affect, physical sensations- is stored in the same form in which it was initially experienced. These memories are stored by association and form memory networks that link present experiences to past experiences and can be triggered by current internal and external stimuli, contributing to present dysfunction [16]. We could not find any EMDR research with the focus of child marriage, trafficking and exploitation is currently lacking. However, one recent study conducted by Jiménez et al, focused on utilizing the EMDR-Protocol for Recent Critical Incidents and Ongoing Traumatic Stress (EMDR-PRECI) with female minors who were survivors of sexual and/or physical abuse with results showing PTSD diagnosis remission in all treatment group participants after treatment [17].

\section{EMDR integrative group treatment protocol for ongoing traumatic stress (EMDR-IGTP-OTS)}

The EMDR-integrative group treatment protocol (EMDR-IGTP) for early intervention was developed by members of the Mexican Association for Mental Health Support in Crisis (AMAMECRISIS) to deal with the extensive need for mental health services after Hurricane Pauline ravaged the coasts of the states of Oaxaca and Guerrero [18] in the year 1997 [18]. The protocol combines the eight EMDR treatment phases with a group therapy model, an art therapy format and uses the Butterfly Hug (BH) as a form of a selfadministered bilateral stimulation [19]. Jarero et al. [20] adapted the EMDR-IGTP to treat older children, adolescents and adults living with ongoing traumatic stress with no post-trauma safety period for memory consolidation and developed the EMDR-IGTP Adapted for Ongoing Traumatic Stress (EMDR-IGTP-OTS) [20,21]. This protocol incorporates many advantages that are suitable for vulnerable populations and various cultures, such as trafficked and exploited women from Bangladesh. The protocol allows for the identification, targeting, and processing of the continuum of multiple traumatic experiences faced by populations who are experiencing ongoing traumatic stress. Individual EMDR treatment can be provided in a group setting to small and large groups in an intensive treatment modality so that patients can receive efficient and effective treatment. All treatment and memory exposure take place in the affect-regulating presence of the therapists. As cross-cultural treatment with cultural sensitivity, EMDR-IGTPOTS reduces cultural resistance to treatment, even to members of reticent cultures, because it is minimally intrusive, and does not require creating a narrative of the traumatic experience, verbal or written disclosure of details, the prolonged reliving of traumatic experience, or homework [22]. The disturbing memory is not visualized mentally as in the standard EMDR protocol, but instead is represented concretely in the participant's drawings or symbols. Relying on drawings or symbols presents a special advantage to provide culturally sensitive and effective treatment for patients who struggle to connect to their cognitive states or 
feel guilty or ashamed; they may be more comfortable expressing their emotional distress through drawing. Also, drawings are used for effective reprocessing with patients with lower levels of literacy, such as the participants in this study [23]. The protocol has been found effective with clients from non-Western cultural backgrounds [22, 24-26].

\section{Objective}

The objective of this study was to evaluate the effectiveness of the EMDR-IGTP-OTS in reducing posttraumatic stress disorder (PTSD), depression, and anxiety symptoms in females who have experienced trauma through living on the streets, child marriage, abandonment by spouse, trafficking, and exploitation in and around Dhaka, Bangladesh through analysis of existing data.

\section{Method}

\section{Study design and ethics}

To measure PTSD, anxiety and depression symptom score changes before and after the provision of the EMDR-IGTPOTS, this field study used a pre-treatment, post-treatment measurement design. The research project was reviewed and approved by Basha's IRB to ensure that the research study was ethically and culturally appropriate for the population. Basha is a privately funded program in Dhaka, Bangladesh that helps women who are mothers and have experienced ongoing or severe trauma including one or more of the following: child marriage, abandonment by spouse, family breakdown, homelessness, sex trafficking, rape, imprisonment, death of spouse or parent, child sex abuse, and formal or informal prostitution. All the women involved in the study were either survivors of trafficking or at high risk of trafficking due to homelessness or destitution.

\section{Participants}

This study was conducted in 2019 at the Basha program office, located in Dhaka, Bangladesh, with women who ranged in age from early 20 s to mid-30s. It is important to note that we do not know the exact age of the women in the study because most of them do not know their exact date of birth or their exact age; only an approximation of age. Participation was voluntary with the participant's verbal and written consent. Appropriate translation was provided for all interviews and during the intervention. A total of 27 women participated in the research project explanation, attended the intake interview, and fulfilled the inclusion criteria.

Inclusion criteria included: (a) females who have been through the same type of ongoing or prolonged traumatic events (e.g., sexual abuse or severe interpersonal violence, such as the women at the Basha program), (b) voluntarily participating in the study, (c) not receiving drug therapy for posttraumatic stress disorder symptoms, and (d) not receiving specialized trauma therapy.
Exclusion criteria included: (a) ongoing self-harm/suicidal or homicidal ideation, (b) diagnosis of schizophrenia, psychotic or bipolar disorder, (c) diagnosis of dissociative disorder, (d) organic mental disorder, (e) current, active chemical dependency problem, (f) significant cognitive impairment (e.g., severe intellectual disability, dementia), and (g) presence of uncontrolled symptoms due to medical illness.

A total of 27 women met the inclusion criteria. Participants ranged in age from mid-20 to mid-30 years old. These ages are estimates as many of the participants did not know their date of birth or their exact age. Grade level of education among participants ranged from no formal education $(n=0)$ to Class $10(\mathrm{n}=10)(\mathrm{M}=2.61)$. The main education system in Bangladesh is divided into the following levels: Primary Level (Class 1-8) and Secondary Level (9-12). There is no middle school system in Bangladesh. It should also be noted that 12 out of the 27 participants reported attempted suicide at some time in their life

\section{Instruments}

We used the Posttraumatic Stress Disorder Checklist for DSM-5 (PCL-5) provided directly by the National Center for PTSD (NCPTSD) and adapted, with the NCPTSD approval for the past week, instead of the past month symptoms to research with a high mobility population $[27,28]$. The instrument was translated and back-translated from English to Bangla. It contains 20 items, including three new PTSD symptoms (compared with the PTSD Checklist for DSM-IV) [29]: blame, negative emotions, and reckless or self-destructive behavior. Respondents indicate how much they have been bothered by each PTSD symptom over the past week (rather than the past month), using a 5-point scale ranging from $0=$ not at all, $1=$ a little bit, $2=$ moderately, 3 = quite a bit, and $4=$ extremely. A total-symptom score of zero to 80 can be obtained by summing the items. The sum of the scores yields continuous measure of PTSD symptoms severity for symptom clusters and the whole disorder Psychometrics for the PCL-5, validated against the Clinician-Administered PTSD Scale-5 (CAPS-5) diagnosis, suggest that a score of 31-33 is optimal to determining a probable PTSD diagnosis, and a score of 33 is recommended for use at pre-set $[28,30]$. The PCL-5 is intended for a variety of clinical and research assessment tasks, including quantifying PTSD symptom severity, measuring the underlying construct of PTSD, establishing a provisional PTSD diagnosis, and estimating the presumed prevalence of PTSD. It is important to mention that at the first assessment time, before answering the PCL-5, all participants were asked to focus specifically on the worst trafficking or exploitative-related experience that currently bothered them the most; then at each subsequent assessment time, they were asked to focus on the same event.

The Hospital Anxiety and Depression Scale (HADS) has been extensively used to evaluate these psychiatric comorbidities in various clinical settings at all levels of healthcare services and 
with general populations $[31,32]$. The instrument was translated and back-translated from English to Bangla. It is a 14 item selfreport scale to measure the anxiety (7 items) and depression (7 items) of patients with both somatic and mental problems using a 4-point Likert scale ranging from 0 to 3 . The response descriptors of all items are Yes, definitely (score 3); Yes, sometimes (score 2); No, not much (score 1); No, not at all (score 0). A higher score represents higher levels of anxiety and depression: a domain score of 11 or greater indicates anxiety or depression; 8-10 indicates borderline case; 7 or lower indicates no signs of anxiety or depression.

\section{Procedure}

\section{Cultural sensitivity, assessment times, collection of data, and confidentiality of data}

To be culturally sensitive, all interviews and protocol administration were conducted in the participant's language (Bangla) by local Bangladeshi university students as trained research assistants. To decrease any possible prejudice against the treatment or the perceived need of treatment because they are "insane," research assistants provided psychoeducation about trauma and answered participant's questions related to trauma, PTSD, anxiety, and depression. Treatment group participants completed the instruments on an individual basis in the different measurement moments. During Time 1, local Bangladeshi university students blind to the research study conducted the intake interview, collected clinical histories, and signed informed consent forms from the participants. Application of instruments was conducted after this procedure by the same research assistants. During Time 2 and Time 3 (post-treatment assessment 30 days after treatment and follow-up assessment 90-days after treatment assessment) measurements were conducted for all participants by the same research assistants blind to the study.

All data was collected, stored, and handled in full compliance with the Guidelines for Good Clinical Practice of the European Medicines Agency (version 1 December 2016) and the Helsinki Declaration as revised in 2013. The participants all gave their verbal and written consent for access to their personal data, which was strictly required for study quality control. All persons involved in this research project are subject to professional confidentiality.

\section{Withdrawal from the Study}

All research participants had the right to withdrawal from the study without justification at any time and with assurances of no prejudicial result. If participants decided to withdraw from the study, they were no longer followed up in the research protocol. There were no withdrawals from this study.

\section{Treatment}

In this study, intensive EMDR therapy was provided [33,34]. Evidence suggests that more frequent scheduling of treatment sessions maximizes PTSD treatment outcomes [35]. This intensive format allowed the participants to complete the full course of treatment in a short period. Participants completed a total of six treatment sessions provided during two consecutive days, three times a day.

\section{Therapists and treatment fidelity}

Provision of the EMDR-IGTP-OTS was provided by one licensed EMDR clinician formally trained in the protocol administration. Three local Bangladeshi social workers, case managers and interpreters collaborated with the clinician. Treatment fidelity and adherence to the protocol was fulfilled by strict observance to all steps of the scripted protocol.

\section{EMDR-IGTP-OTS treatment description and tolerance}

An individual history-taking and assessment session was conducted for each potential group member to determine their suitability and readiness for EMDR treatment. Treatment was supervised by an EMDR Certified Therapist / EMDRIA Approved Consultant. Each of the participants received an average of six hours of treatment, provided during six group treatment sessions, three times daily during two consecutive days at the Basha program office in Dhaka, Bangladesh. EMDR-IGTP-OTS treatment focused only on the distressing memories related to their experiences with exploitation, trafficking or child marriage and did not address any other memories. During this process, participants followed directions from the team leader and worked quietly and independently on their distressing memories. The first treatment session lasted 90 minutes. Subsequent five treatment sessions lasted an average of 45 minutes. Time for rest in between sessions lasted an average of one hour. Activities during rest time included brief physical activity, socializing and teatime.

To encompass the whole ongoing traumatic stress spectrum, the team leader asked each of the participants to "run a mental movie of everything that happened just before they were exploited, trafficked or married until now, or even looking into the future." The initial treatment target was the most distressing moment in the mental movie. In subsequent sessions, the team leader asked patients to run the mental movie again and then to target any memory that was currently disturbing, noticing associated emotions and body sensations. Participants in this study used the Butterfly Hug (BH) 36 times as a self-administered bilateral stimulation method to process traumatic material [19]. During the $\mathrm{BH}$, patients are instructed to stop when they felt in their body that is had been enough. The instruction allows for enough sets of bilateral stimulation (BLS) for processing the traumatic material and helps to regulate the stimulation to maintain the patients in their window of tolerance allowing for appropriate reprocessing $[36,37]$. All participants had the opportunity to reprocess more than one memory. No adverse effects were reported during treatment, at one-month post-treatment assessment, or at 90days follow-up assessment. 


\section{Statistical Analysis}

Anxiety, depression and PTSD symptoms were measured, and statistical analysis was conducted for all participants who fulfilled the inclusion criteria at the beginning of the study and who completed the post-treatment and follow-up assessments. Analyses of variance (ANOVA) for repeated measurements were used for PTSD, anxiety and depression symptoms; $t$ test and Cohen's d effect size were calculated using within and between designs for the different mean comparisons.

\section{Results}

All 27 participants were included in the statistical analysis. The ANOVA results of the HADS-anxiety [31] scores show that the Anxiety measures were significantly different between tests, $F(2,42)=3.62, p=.035$ (Figure 1). After conducting t-tests across different measures with Bonferroni correction, we found no significant differences between the pre-test and Day-30 test as well as between the Day-30 test and Day-90 test, p>.05; however, a significant difference was found between the pre-test and Day90 test, $\mathrm{p}=0.019$. Therefore, the null hypothesis is rejected in favor of the hypothesis that EMDR-IGTP-OTS lowers the anxiety symptoms of participants according to the HADS Anxiety [31]. The effect size between the pre-test and Day-30 test is 0.09 , which is small; the effect size between the Day-30 test and Day-90 test is 0.39 , which is medium; the effect size between the pre-test and Day-90 test is 0.66 , which is a substantial finding.

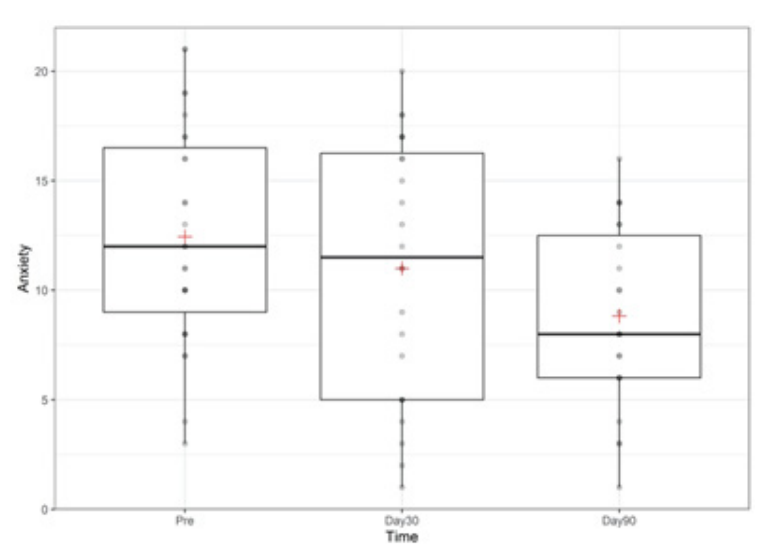

Figure 1: Anxiety Scores (HADS).

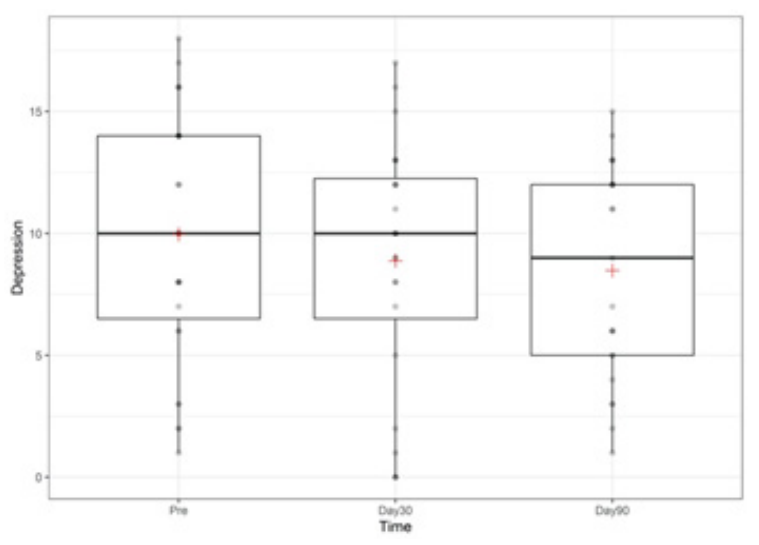

Figure 2: Depression Scores (HADS).

In comparison between the pre-test and Day-30 test, we saw decreasing patterns in general (see Pairs 1, 2, and 3). The decreasing patterns continued up to Day 90 (see Pairs 4, 5, and 6). After conducting paired t-tests, we found significant differences for Pairs 3, 5, and 6; the mean of PCL-5 [38] in the pre-test was significantly different from that in the Day-30 test, $\mathrm{p}=.001$; the mean of HADS-Anxiety [31] in the pre-test significantly differed from that in the Day-90 test, p=.006; the mean of PCL-5 [38] in the pre-test was significantly different from that in the Day-90 test, $p=.001$. Due to these significant findings, we reject the null hypothesis. Note that due to missing values in measures, the sample sizes for each t-test were not the same. See Figures 1- 3 
below. The participants live in the city of Dhaka (or the outskirts of the city) and travel to the Basha program regularly to work each day. Participants in this study are female, and range in age from early 20's to late 30's. Many of these women had faced exploitation, abuse, and abandonment by spouses and parents. They often suffer PTSD, anxiety and depression symptoms due to these horrific experiences. Challenges for the study included having a quiet space in which to hold the session in as construction work on the interior of the building created a very loud environment.

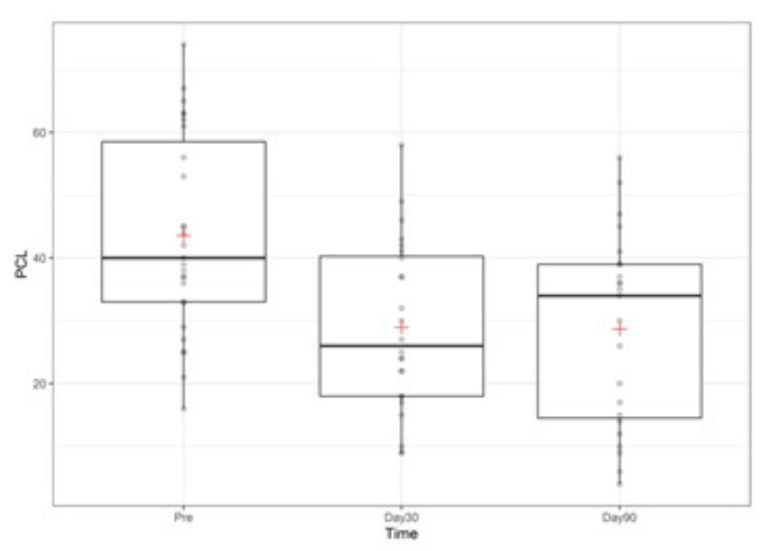

Figure 3: Posttraumatic Stress Disorder Checklist for DSM-5 Scores (PCL-5).

\section{Study Discussion}

The main objective of this study was to evaluate the effectiveness of the EMDR-integrative group treatment protocol for ongoing traumatic stress in reducing posttraumatic stress disorder (PTSD), depression, and anxiety symptoms in women who have experienced exploitation, trafficking and/or early marriage.

The 27 participants in the research study, reprocessed their exploitation, trafficking and/or early marriage-related traumatic memories with efficiency (only six group sessions during two consecutive days, three times a day), and efficacy, as we can see in the differences between pre-and post-treatment scores for the PCL-5 and HADS. Those scores showed a significant improvement in PTSD, anxiety and depression symptoms for all participants.

The results show that the HADS-Depression [31] measures were significantly different between tests, $F(1.54,32.34)=0.96$, $\mathrm{p}>.05$ (Figure 2). Note that since we violate the assumption of sphericity i.e., the variances of the differences between measures, we use Greenhourse-Geisser correction for F tests. After conducting t-tests across different measures with Bonferroni correction, we found no significant differences, $p>.05$, therefore we accept the null hypothesis. The effect sizes for two comparisons are small: 0.15 between the pre-test and Day- 30 test as well as 0.12 between the Day-30 test and Day-90 test; the effect size between the pretest and Day-90 test is 0.41 .

The ANOVA results show that the PCL-5 [38] measures were significantly different between tests, $F(2,42)=8.45, p=.001$ (Figure 3). After conducting t-tests across different measures with Bonferroni correction, we found significant differences between the pre-test and Day-30 test, $\mathrm{p}=.005$, as well as between the pre- test and Day-90 test, $\mathrm{p}=.005$; however, no significant difference was found between the Day-30 test and Day-90 test, $p>.05$. The effect sizes between the pre-test and Day-30 test and between the pre-test and Day-90 test are 0.61, which is large, while the effect size between the Day-30 test and Day-90 test is 0.14 , which is small. Though there is no significant difference found in scores between the Day- 30 test and Day- 90 test, the significance differences between the pre-test and Day-30 test, $p=.005$, as well as between the pre-test and Day- 90 test, $\mathrm{p}=.005$ support the original hypothesis that the EMDR-IGTP-OTS lowers PTSD symptoms in participants according to the PCL-5 [37], and the null hypothesis is rejected.

\section{Conclusion}

Studies evaluating the efficacy of psychological treatments for PTSD, anxiety, and depression carried out for women who have survived exploitation, trafficking, sexual assault and abuse, and/ or early marriage are rare and greatly needed. Not only have these women been exploited and abused, but they are also neglected by existing research. The problem therein lies in the lack of research and treatment options to serve this large and unserved population. This study highlights the need for mental health services within this population. The study results indicate that with the EMDR-IGTP-OTS, organizations could have the ability to scale up treatment to serve the masses through an intensive, group intervention that can serve many women at one time. The results of this research show that the EMDR-IGTP-OTS could be an important component of a multidisciplinary approach to reducing or eliminating PTSD, anxiety and depression symptoms in trafficked and exploited women in Bangladesh. This modality is suggested as an effective intervention to help this vulnerable population. 


\section{Psychology and Behavioral Science International Journal}

\section{Implications}

\section{For counselors}

This study shows how the EMDR-IGTP-OTS can be used with large, medium or small groups of women worldwide: those who have faced exploitation and abuse. EMDR-IGTP-OTS is a suggested treatment modality to be used with clients that have been through similar experiences and are suffering from PTSD and anxiety symptoms. Clinically, the EMDR-IGTP-OTS can be used with clients who have a PTSD diagnosis. It is important to note that a counselor must be EMDR trained in order to appropriately employ the EMDR-IGTP-OTS. Counselor training can be obtained by attending a seminar, either online or in-person, in order to be qualified to use the modality. Scaling Up provides many trainings every year at various locations all over the country. Learning this modality and protocols will enhance clinicians who work with exploited women, as well as those who have experienced ongoing traumatic stress in many other circumstances.

\section{For counselor educators}

EMDR-IGTP-OTS effectiveness has been demonstrated through many studies, suggesting its value for inclusion in counselor education. It is suggested that EMDR-IGTP-OTS research is studied as part of group and/or trauma curricula as students are also learning about how to treat PTSD (included with the AIP model and EMDR). Counselor education students should be encouraged to include this modality in their research papers, to learn about the AIP model as it relates to group treatment, and to consider the usefulness of EMDR-IGTP-OTS in the work of humanitarian aid, relief aid, and overseas treatment and research. Scaling Up accepts masters' level and PhD level counselor educators as research interns, and students should consider taking advantage of this program as they complete their programs [39].

\section{Limitations and Future Directions}

This study has several limitations, notably the pretreatment and posttreatment measurement design without a control group and the small sample size. Future research with randomized controlled clinical trials with larger samples using an instrument to conduct formal PTSD diagnosis (e.g., CAPS-5), with follow-up at six or twelve months when possible to evaluate long-term effect in participants is highly recommended.

\section{Conflict of Interest and Founding}

The authors have no relevant financial interest or affiliation with any commercial interest related to the subjects discussed in the article. This research received no specific grant from any funding agency, commercial or not-for-profit sectors.

\section{Acknowledgement}

We want to express gratitude to the following individuals for their contributions: The women, staff and research assistants at Basha and Dr. Ignacio Jarero.

\section{References}

1. UNICEF (2018) Statistics pulled from Child marriage around the world.

2. UNICEF (2018) Child marriage.

3. Amin, Sajeda, Jyotirmoy Saha, Masuma Billah, Nargis Sultana, et al. (2018) Accelerating action to end child marriage in Bangladesh. New York and Bangladesh: Population Council, USA.

4. Nasrullah M, Zakar R, Zakar MZ (2014) Child marriage and its associations with controlling behaviors and spousal violence against adolescent and young women in Pakistan. Journal of Adolescent Health 55(6): 804-809.

5. Gage AJ (2013) Association of child marriage with suicidal thoughts and attempts among adolescent girls in Ethiopia. Journal of Adolescent Health 52(5): 654-656.

6. Le Strat Y, Dubertret C, Le Foll B (2011) Child marriage in the United States and its association with mental health in women. Pediatrics 128(3): 524-530.

7. Efevbera Y, Bhabha J, Farmer PE, Fink G (2017) Girl child marriage as a risk factor for early childhood development and stunting. Social Science \& Medicine 185: 91-101.

8. Maertens A (2013) Social norms and aspirations: Age of marriage and education in rural India. World Development 47: 1-15.

9. Suarez PA (2018) Child-bride marriage and female welfare. European Journal of Law and Economics. 45(1): 1-28.

10. Yount KM, Crandall A, Cheong YF, Osypuk TL, Bates LM, et al. (2016) Child marriage and intimate partner violence in rural Bangladesh: A longitudinal multilevel analysis. Demography 53(6): 1821-1852.

11. Vogl T (2010) Sisters, Schooling, and Spousal Search: Evidence from South Asia. Paper presented at Northeast Universities Development Consortium Conference on 6-6 November 2010 at MIT.

12. Shapiro F (2018) Eye movement desensitization and reprocessing. Basic principles, protocols, and procedures. ( $3^{\text {rd }}$ edn), Guilford Press, United States.

13. Diagnostic and statistical manual of mental disorders (2013) American Psychiatric Association (5 $5^{\text {th }}$ edn). American Psychiatric Publishing, Arlington, USA.

14. Bisson JI, Ehlers A, Matthews R, Pilling S, Richards D, et al. (2007) Psychological treatments for chronic post-traumatic stress disorder: Systematic review and meta-analysis. British Journal of Psychiatry 2: 97-104.

15. Rodenburg R, Benjamin A, De Roos C, Meijer AM, Stams GJ (2009) Efficacy of EMDR in children: A meta-analysis. Clinical Psychology Review 29: 599-606.

16. Jarero I, Artigas L (2018) AIP model-based Acute Trauma and Ongoing Traumatic Stress Theoretical Conceptualization (Second Edition). Iberoamerican Journal of Psycho traumatology and Dissociation 10(1): $1-7$.

17. Jiménez G, Becker Y, Varela C, García P, Amparo Nuño M, et al. (2020) Multicenter Randomized Controlled Trial on the Provision of the EMDR-PRECI to Female Minors Victims of Sexual and/or Physical Violence and Related PTSD Diagnosis. American Journal of Applied Psychology 9(2): 42-45.

18. Jarero I, Artigas L (2009) EMDR integrative group treatment protocol. Journal of EMDR Practice \& Research 3(4): 287-288.

19. Artigas L, Jarero I (2014) The Butterfly Hug. In: M Luber (Ed.), Implementing EMDR Early Mental Health Interventions for Man-Made and Natural Disasters. Springer, New York, USA, pp. 127-130. 
20. Jarero I, Artigas L, Uribe S, García LE, Cavazos MA, et al. (2015) Pilot Research Study on the Provision of the EMDR Integrative Group Treatment Protocol with Female Cancer Patients. Journal of EMDR Practice and Research 9(2): 98-105.

21. Jarero I, Artigas L, Uribe S, García LE (2016) The EMDR Integrative Group Treatment Protocol for Patients with Cancer. Journal of EMDR Practice and Research 10(3): 199-207.

22. Hurn R, Barron I (2018) The EMDR Integrative Group Treatment Protocol in a Psychosocial Program for Refugee Children: A Qualitative Pilot Study. Journal of EMDR Practice and Research 12(4): 208-223.

23. Shapiro F (2016) Clinician's Corner: EMDR Therapy. International Society for Traumatic Stress Studies. Stress Points.

24. Nickerson M (2017) Cultural Competence and Healing Culturally Based Trauma with EMDR Therapy: Innovative Strategies and Protocols $\left(1^{\text {s }}\right.$ edn). Springer Publishing LLC, New York, USA, pp. 3-28.

25. Wilson S, Thinker R, Becker L, Hofmann A, Kleiner K (2000) A field study of EMDR with Kosovar-Albanian refugee children using a group treatment protocol. Symposium presented at the annual conference of the EMDR International Association, Toronto, Canada.

26. Perilli S, Giuliani A, Pagani M, Mazzoni GP, Maslovari G, et al. (2019) EMDR Group Treatment of Children Refugees -A Feasibility Field Study. Journal of EMDR Practice and Research 13(3): 143-155.

27. Weathers FW, Litz BT, Keane TM, Palmieri PA, Marx BP, et al. (2013) The PTSD Checklist for DSM-5 (PCL-5). Scale available from the National Center for PTSD.

28. Bovin MJ, Marx BP, Weathers FW, Gallagher MW, Rodriguez P, et al. (2016) Psychometric properties of the PTSD checklist for diagnostic and statistical manual of mental disorders-Firth edition (PCL-5) in veterans. Psychol Assess 28(11): 1379-1391.

29. Diagnostic and statistical manual of mental disorders (2000) ( $4^{\text {th }}$ edn) American Psychiatric Association. Arlington, Virginia, USA.
30. Weathers FW, Blake DD, Schnurr PP, Kaloupek DG, Marx BP, et al. (2013) Clinician-administered PTSD Scale for DSM-5. National Center for Posttraumatic Stress Disorder, Boston, USA.

31.Zigmond AS, Snaith RP (1983) The Hospital Anxiety and Depression Scale. Acta Psychiatrica Scandinavica 67: 361-370.

32. Ying Lin C, Pakpour AH (2017) Using Hospital Anxiety and Depression Scale (HADS) on patients with epilepsy: Confirmatory factor analysis and Rasch models. Seizure (45): 42-46.

33. Hurley EC (2018) Effective Treatment of Veterans With PTSD: Comparison Between Intensive Daily and Weekly EMDR Approaches. Front Psychol 9: 1458.

34. Bongaerts H, Van Minnen A, de Jongh A (2017) Intensive EMDR to treat patients with complex posttraumatic stress disorder: A case series. Journal of EMDR Practice and Research 11(2): 84-95.

35. Gutner CA, Suvak MK, Sloan DM, Resick PA (2016) Does timing matter? Examining the impact of session timing on outcome. Journal of Consulting and Clinical Psychology 84: 1108-1115.

36. Ogden P, Minton K, Pain C (2006) Trauma and the body: A sensorimotor approach to psychotherapy. Norton, New York, USA.

37. Siegel DJ (1999) The developing mind: How relationships and the brain interact to shape who we are. Guilford Press, New York, USA.

38. Blevins CA, Weathers FW, Davis MT, Witte TK, Domino JL (2015) The posttraumatic stress disorder checklist for DSM-5 (PCL-5): Development and initial psychometric evaluation. Journal of traumatic stress 28(6): 489-498.

39. Walsh SF (2020) The EMDR Integrative Group Treatment Protocol for Ongoing Traumatic Stress with Female Survivors of Child Marriage, Trafficking, and Exploitation in Dhaka, Bangladesh.

\section{Your next submission with Juniper Publishers will reach you the below assets}

- Quality Editorial service

- Swift Peer Review

- Reprints availability

- E-prints Service

- Manuscript Podcast for convenient understanding

- Global attainment for your research

- Manuscript accessibility in different formats

( Pdf, E-pub, Full Text, Audio)

- Unceasing customer service

Track the below URL for one-step submission https://juniperpublishers.com/online-submission.php 